Claremont Colleges

Scholarship@ Claremont

CGU Faculty Publications and Research

CGU Faculty Scholarship

1-1-2009

\title{
Affective Decision-making Predictive of Chinese Adolescent Drinking Behaviors
}

\author{
Lin Xiao \\ University of Southern California \\ Antoine Bechara \\ University of Southern California \\ Jerry L. Grenard \\ Claremont Graduate University \\ Alan W. Stacy \\ Claremont Graduate University \\ Paula Palmer \\ Claremont Graduate University \\ See next page for additional authors
}

\section{Recommended Citation}

Xiao, L., Bechara, A., Grenard, J.L., Stacy, A.W., Palmer, P., Wei, Y., Jia, Y., Fu, X., \& Johnson, C.A. Affective decision-making predictive of Chinese adolescent drinking behaviors. J Int Neuropsychol Soc, 15(4), 547-57, 2009.

This Article is brought to you for free and open access by the CGU Faculty Scholarship at Scholarship @ Claremont. It has been accepted for inclusion in CGU Faculty Publications and Research by an authorized administrator of Scholarship @ Claremont. For more information, please contact scholarship@cuc.claremont.edu. 


\section{Authors}

Lin Xiao, Antoine Bechara, Jerry L. Grenard, Alan W. Stacy, Paula Palmer, Yonglan Wei, Yong Jia, Xiaolu Fu, and C. Anderson Johnson 


\title{
Affective decision-making predictive of Chinese adolescent drinking behaviors
}

\author{
LIN XIAO, ${ }^{1}$ ANTOINE BECHARA, ${ }^{1}$ L. JERRY GRENARD,${ }^{2} \mathrm{~W}$. ALAN STACY $, 3,4$ PAULA PALMER, $, 3,4$ \\ YONGLAN WEI, ${ }^{5}$ YONG JIA, ${ }^{5}$ XIAOLU FU, ${ }^{5}$ AND C. ANDERSON JOHNSON ${ }^{3,4}$ \\ ${ }^{1}$ Department of Psychology, Brain and Creativity Institute, University of Southern California, Los Angeles, California \\ ${ }^{2}$ Department of Health Services, UCLA School of Public Health, Los Angeles, California \\ ${ }^{3}$ Institute for Health Promotion and Disease Prevention Research, University of Southern California, Los Angeles, California \\ ${ }^{4}$ School of Community and Global Health, Claremont Graduate University, Claremont, California \\ ${ }^{5}$ Chengdu Municipal Center for Disease Control and Prevention, Chengdu, China
}

(Received December 23, 2008; Final Revision March 24, 2009; Accepted March 25, 2009)

\begin{abstract}
The goal of the current investigation was to address whether affective decision making would serve as a unique neuropsychological marker to predict drinking behaviors among adolescents. We conducted a longitudinal study of 181 Chinese adolescents in Chengdu city, China. In their 10th grade (ages 15-16), these adolescents were tested for their affective decision-making ability using the Iowa Gambling Task (IGT) and working memory capacity using the Self-Ordered Pointing Test. Self-report questionnaires were used to assess academic performance and drinking behaviors. At 1-year follow-up, questionnaires were completed to assess drinking behaviors, and the UPPS Impulsive Behavior Scale was used to examine four dimensions of impulsivity: urgency, lack of premeditation, lack of perseverance, and sensation seeking. Results indicated that those adolescents who progressed to binge drinking or exhibited consistent binge drinking not only performed poorly on the IGT but also scored significantly higher in urgency compared to those who never or occasionally drank. Moreover, better IGT scores predicted fewer drinking problems and fewer drinks 1 year later after controlling for demographic variables, the previous drinking behaviors, working memory, and impulsivity. These findings suggest that deficits in affective decision making may be important independent determinants of compulsive drinking and potentially addictive behavior in adolescents.
\end{abstract} (JINS, 2009, 15, 547-557.)

Keywords: Executive function, Affective control, Impulsivity, Working memory, Binge drinking, Iowa Gambling Task

\section{INTRODUCTION}

A considerable body of evidence has accumulated a variety of risk factors for escalation of drinking and alcohol use problems in late adolescence. These factors range from behavioral and psychological levels to neurocognitive levels such as drinking behaviors in the earlier development stage, impulsivity, and poor executive functions (EFs) (Brown et al., 2008; Nigg et al., 2006). In this study, we investigate the effect of two specific EFs, affective decision making and working memory, on the progression of adolescents' drinking

Work was performed in the Institute for Health Promotion and Disease Prevention Research, University of Southern California, Los Angeles, CA.

Correspondence and reprint requests to: C. Anderson Johnson, School of Community and Global Health, Claremont Graduate University, 180 E. Via Verde, Suite 100, San Dimas, California 91773. E-mail: andy.johnson@ cgu.edu behaviors. Specifically, we examine whether affective decision making could serve as a unique neuropsychological marker predictive of the change in drinking behaviors among adolescents, independent of other behavioral and psychological functions.

Deficits in a wide range of EF processes including response inhibition, working memory, attentional control, abstract reasoning, cognitive flexibility, and planning have been identified in the research on alcohol use among adolescents, and most of these processes have been linked to neural activity within the dorsolateral sector of the prefrontal cortex (DLPC) (Grenard et al, 2008; Iacono et al., 1999; Nigg et al., 2006; Thush \& Wiers, 2008). Little research has addressed the neuropsychological processes in adolescent alcohol use, which may rely heavily on the orbital/ventromedial sector of the prefrontal cortex (OFC/VMPC) (Overman et al., 2004). Studies of humans with OFC/VMPC brain injury and neuroimaging studies suggest that this region is highly relevant for processing 
emotional experience associated with rewards and punishments (O'Doherty et al., 2001; Oya et al., 2005). Studies also indicate that maturation of the OFC/VMPC, especially the frontal pole (e.g., Brodmann's area 10), might be a developmentally distinct process from that of other regions of the frontal lobe, and OFC/VMPC-related neuropsychological functions might mature later than do those mediated by the DLPC (Crone \& van der Molen, 2004; Hooper et al., 2004). Therefore, it is important to investigate OFC/VMPC-related brain functions among adolescents' reward-seeking behaviors such as alcohol consumption.

One of the most important neuropsychological processes mediated by OFC/VMPC-related circuitry is affective decision making or decision making that requires appraisal of the emotional or motivational significance of stimuli (e.g., their potential for rewards and punishments) (Kerr \& Zelazo, 2004). One neuropsychological task designed to engage these affective decision-making processes in the brain is the Iowa Gambling Task (IGT), which resembles real life in the way it factors reward, punishment, and uncertainty about future outcomes (Bechara et al., 1994). Compared to other tasks, which assess brain functions related to explicit calculation of probabilities or expected value, the IGT requires participants to learn implicitly from their past experience (such as rewards and punishments encountered during the task) in order to infer the probable outcomes of the choices they are currently making (Bechara, 2004). Studies show that the learning processes involved in the IGT are strongly influenced by affective and emotional systems (Stocco \& Fum, 2008; Wagar \& Dixon, 2006). One previous study also showed that normal controls can develop anticipatory somatic "warning" markers preceding disadvantageous choices when they play the IGT task. However, this warning signal is missing for patients who have damage to the OFC/VMPC (Bechara et al., 1996).

The current study tests the ability of affective decision making to account for changes in adolescents' drinking behaviors at a 1-year follow-up. We also take into account other risk factors reported in previous studies including previous drinking behaviors, working memory capacity, and impulsivity. Our aim was to test the hypothesis that affective decision making would emerge as a unique neuropsychological marker that predisposes individuals to compulsive, binge drinking, and problem drinking behavior in adolescents. We tested this hypothesis via two statistical methods. First, we categorized these adolescents according to their drinking behaviors at two different points in time (baseline and 1 year later), and we predicted that those who progressed to binge drinking or exhibited consistent binge drinking would perform poorly on the IGT compared to never or occasional drinkers. Second, we employed linear models to test the prediction that even after controlling for potential confounders (demographic variables, previous drinking behaviors, working memory capacity, and impulsivity), adolescents with poor decision-making capabilities would be more likely to escalate over time in the number of drinks taken in a single drinking episode and in drinking-related problems. By contrast, individuals with better decision-making capabilities would be more resistant to these behavioral problems in drinking.

\section{METHODS}

\section{Sample}

Data collection for this study was supported by the Pacific Rim Transdisciplinary Tobacco and Alcohol Use Research Center, which is investigating social, environmental, and biological determinants of tobacco and alcohol use and abuse among youth in China (C.A.J., Principle Investigator). All research protocols and instruments were approved by the University of Southern California and Chengdu, China Centers for Disease Control and Prevention institutional review boards. To ensure maximum variability across the student sample, two academic high schools, one of high and one of low/middle academic status, and two vocational schools, one of middle and one of low academic status, were selected for the study. One 10th-grade class from each of the four schools was randomly selected, and a total of 223 students were invited to participate. Students voluntarily took part in the study and were told that they could discontinue their participation at any time. Out of the total, 16 participants at Time 1 and 26 in the 1-year follow-up (Time 2) were excluded from the data analysis due to computer malfunctions or failure to complete the survey or follow instructions on the Self-Ordered Pointing Test (SOPT). The analytic data set included 181 participants $(81.2 \%$ of total participants).

\section{Measures and Procedures}

Baseline (Time 1) measures included two computer-assisted neurocognitive assessments and a paper-and-pencil self-report questionnaire. One-year follow-up (Time 2) measures only included a paper-and-pencil self-report questionnaire. The instructions for the neuropsychological tasks and the questionnaires were translated into Mandarin Chinese (the only language used in the surveys) and back-translated prior to use. The questionnaires incorporated items from the U.S. Centers for Disease Control and Prevention's (1999) Behavioral Risk Factor Surveillance System and Youth Risk Behavior Surveillance Survey (Kolbe et al., 1993). To ensure that each item captured the proper idiomatic language, each item was reviewed for consensus by a group of English- and Chinesespeaking researchers and graduate students. We used focus groups and interviews with the local teachers and students to assess the appropriateness and feasibility for the actual questionnaires. The questionnaires have also been used in our previous large-scale longitudinal China Seven Cities Study (Johnson et al., 2006).

\section{Time 1}

Neuropsychological assessments. Iowa Gambling Task (IGT). As described in previous studies (Bechara et al., 1994), the IGT is a computerized version of the gambling task with an automated and computerized method for collecting data. In the IGT, four decks of cards labeled A', B', C', and D' are displayed on the computer screen. The backs of the cards resemble real decks of cards. The participant starts the task 
with a sum of make-believe money in his or her account, represented by a green bar that changes in length as the participant "wins" or "loses" money during the task. The subject is required to select one card at a time from one of the four decks. When the subject selects a card, a message indicating the amount of money the subject has won or lost is displayed on the screen. The preprogrammed schedules of gain and loss are controlled by the computer. Turning each card can bring an immediate large reward in decks A' and B' and a small reward in decks C' and D'. As the game progresses, there are also unpredictable losses among the cards. Total losses are much larger in every 10 cards in decks A' and B' compared to the losses in decks C' and D'. Decks A' and B' are equivalent in terms of overall net losses, and decks C' and D' are equivalent in terms of overall net gains over the course of the trials. The difference is that in decks A' and C', the punishments are more frequent but are of smaller magnitude. Whereas in decks B' and D', the punishments are less frequent but are of greater magnitude. Thus, decks A' and B' are disadvantageous because they yield high immediate gains but greater losses in the long run, and decks C' and D' are advantageous in that they yield lower immediate gains but smaller losses in the long run. An IGT net score is obtained by subtracting the total number of selections from the disadvantageous decks ( $\left.\mathrm{A}^{\prime}+\mathrm{B}^{\prime}\right)$ from the total number of selections from the advantageous decks $\left(C^{\prime}+D^{\prime}\right)$.

Self-Ordered Pointing Test. We used a computerized version of the SOPT (Peterson et al., 2002), which was based on a task originally developed by Petrides and Milner (1982). This task tests the capacity for transient online storage (Perry et al., 2001) and for active monitoring and retrieval of increasing amounts of information held in working memory (Petrides, 1995). It has been linked to neural activity within the DLPC (Petrides et al., 1993) and has been found to be effective in diverse adolescent populations (Chaytor \& Schmitter-Edgecombe, 2004; Thush et al., 2008). The SOPT has both verbal and nonverbal components with three trials of each. In the verbal component, subjects view pictures of concrete nameable objects (clock, book, bus, etc.), whereas in the nonverbal component, subjects view abstract designs that are difficult to name or encode verbally. In each trial, 12 pages are presented sequentially, with each page depicting the same 12 pictures but in a different spatial arrangement on each page. Subjects are instructed to point to a different picture in each presentation. To effectively select a different picture each time, subjects must retain pictures in working memory. The total number of correct selections of different pictures represents the working memory score. There is a maximum possible score of 12 on each trial and a total of 72 for all six trials. In our study, the internal consistency across the six trials was .86 .

Questionnaires. Drinking behaviors. Four drinking categories of participants were identified: (a) never drinkers, who were defined as those who reported never having had one drink of alcohol in their life; (b) ever drinkers, who were defined as those who had had at least one drink of alcohol in their life but not in the past 30 days; (c) past 30-day drinkers, who were defined as those who had had at least one drink of alcohol in the past 30 days but did not consume four or more drinks of alcohol in a row in the past 30 days; and (d) binge drinkers, who were defined as those who had had four or more drinks of alcohol in a row on at least one occasion in the past 30 days.

Number of drinks taken in a single drinking episode. was assessed using the following item: "When you drink alcohol, how many drinks do you usually have?" The six response options ranged from "I don't drink alcohol" to "5 or more." The response "I don't drink alcohol" was assigned a score of "0."

Drinking problems. were assessed using the Rutgers Alcohol Problem Index (RAPI) (White \& Labouvie, 1989). Some example questions included "(because of drinking) I got into fights with other people (friends, relatives, strangers)"; "I went to school drunk." Each response was assigned a score of " 1 " or " 0 " depending on the response. The score for drinking problems was the sum of the 23 items. The Cronbach's alpha of the RAPI scale was .88 .

School academic performance. was assessed by the following question: "What is your usual academic performance at your current school or the last school where you received grades?" The five response options ranged from "mostly A's, or 90 or more points, or superior" to "mostly F's, or below 60 points, or failing." A higher score represented a higher academic performance.

\section{Time 2}

Questionnaires. Drinking behaviors. The same questions in the baseline were used to ask drinking behaviors. In order to better demonstrate the neuropsychological performance and development of drinking behaviors among adolescents, we categorized the participants according to their drinking behaviors in the 2 years that we tested as shown in Table 1 . Those who were never drinkers at both Time 1 and Time 2 were grouped as consistent never drinkers. Those who were never, ever, or past 30-day drinkers at Time 1 and were ever or past 30-day drinkers at Time 2 were grouped as occasional drinkers. Those who were never, ever, or past 30-day drinkers at Time 1 and progressed to binge drinkers at Time 2 were new binge drinkers. Those who were binge drinkers at Time 1 but became ever or past 30-day drinkers at Time 2 were grouped as quitters. Those who were binge drinkers at both years were grouped as consistent binge drinkers.

Impulsivity. was assessed using UPPS Impulsive Behavior Scale (Whiteside \& Lynam, 2001). The scale consists of 45 items evaluating four different facets of impulsivity: the urgency (12 items) measuring an individual's tendency to give in to strong impulses, specifically when accompanied by negative emotions such as depression, anxiety, or anger (e.g., "When I am upset I often act without thinking."); the premeditation (11 items) measuring an individual's ability to think through the potential consequences of his or her behavior 
Table 1. Drinkers stratification

\begin{tabular}{llcl}
\hline \hline \multicolumn{2}{c}{ Drinkers } & & \\
\cline { 1 - 2 } Time 1 & \multicolumn{1}{c}{ Time 2 } & $\%(N)$ & Groups with different drinking trajectories \\
\hline Never & Never & $32.0(58)$ & Consistent never drinkers \\
Never/ever/past 30 days & Ever/past 30 days & $50.3(91)$ & Occasional drinkers \\
Never/ever/past 30 days & Binge & $6.6(12)$ & New binge drinkers \\
Binge & Ever/past 30 days & $5.0(9)$ & Quitters \\
Binge & Binge drinkers & $6.1(11)$ & Consistent binge drinkers \\
Total & & $100(181)$ & \\
\hline \hline
\end{tabular}

before acting (e.g., "I like to stop and think things over before I do them."); the perseverance (10 items) measuring an individual's ability to persist in completing jobs or obligations despite boredom and/or fatigue (e.g., "I generally like to see things through to the end."); and the sensation seeking (12 items) measuring an individual's preference for excitement and stimulation (e.g., "I generally seek new and exciting experiences and sensations."). Items of the scale are scored from 1 (I agree strongly) to 4 (I disagree strongly). Some items were reversed in such a way that a high score reveals an impulsivity trait of personality. The Cronbach's alpha of the UPPS Impulsive Behavior Scale was .82 for premeditation, .87 for urgency, .88 for sensation seeking, and .80 for perseverance. The total impulsivity score was the sum of the four subscales.

\section{Data Analysis}

Data were analyzed with the Statistical Package for the Social Sciences for Windows, Version, 15.0 (SPSS Inc., Chicago, IL). Among groups with different drinking trajectories, chi-square tests were used to test for differences in frequency distributions by gender and school type, and one-way analysis of variance (ANOVA) tests were used to test for differences in means of age, working memory, school academic performance, and impulsivity. To analyze the IGT performance profile, we conducted between-within ANOVA tests with "Block" as within-subject factor. Associations among neuropsychological performance, school academic performance, and impulsivity were calculated as partial correlations (controlling for age, gender, and school type). To reveal the unique contributions of affective decision making to Year 1 drinking behaviors, we tested a set of hierarchical regression equations with baseline values of the dependent measure, demographic characteristics, working memory capacity, academic performance, and impulsivity entered in Step 1 and the IGT net score entered in Step 2 to predict Year 1 alcohol-related outcomes.

\section{RESULTS}

\section{Demographic Characteristics}

The demographic characteristics of the groups with different drinking trajectories are shown in Table 2. Most participants were 16 years old at Time 1 , and there was no significant difference in age among groups $(p>.05)$. Compared to females, there were significantly more males in the new binge drinker and consistent binge drinker groups $\left(\chi^{2}{ }_{(4)}=11.5 ; p<\right.$ $.05)$. Compared to the academic school, there were marginally significantly more vocational school students in the new binge drinker and consistent binge drinker groups $\left(\chi^{2}(1)=\right.$ 3.6; $p=.06)$.

One-way ANOVA tests revealed that the number of drinks taken in a single drinking episode and drinking problems at Time 1 were significantly different among groups of drinkers with different drinking trajectories across Time 1 and Time 2 $\left(F_{(4,176)}=24.7, p<.001 ; F_{(4,176)}=11.6, p<.001\right.$, respectively). Post hoc Tukey's tests showed that quitters and consistent binge drinkers had more drinks and more drinking problems than other groups $(p<.05)$. However, there was no differences in working memory capacity and school academic performance among the groups of drinkers $(p>.1)$.

New and consistent binge drinkers took more drinks and experienced more drinking problems at Time 2 than other groups $(p<.05)$. One-way ANOVA tests revealed significant difference in impulsivity at Time 2 among different groups $\left(F_{(4,176)}=2.45 ; p<.05\right)$. Post hoc Tukey's tests demonstrate that the consistent binge drinkers and new binge drinker scored higher in impulsivity than never and occasional drinkers $(p<.05)$. Among four facets of impulsivity, new and consistent binge drinkers did not differ from others in sensation seeking, (lack of) perseverance, and (lack of) premeditation. However, they showed higher score in urgency than never and occasional drinkers and other drinkers $(p<.05)$.

\section{Behavioral Performance on the IGT at Time 1}

We plotted the IGT net scores across five blocks of 20 cards each in Figure 1 (left plot: consistent never drinkers, occasional drinkers, and new binge drinkers; right plot: quitters and consistent binge drinkers). As the task progressed, never and occasional drinkers showed similar learning curves, and they gradually switched their preferences toward the advantageous decks (C' and D') and away from the disadvantageous decks (A' and B'), as reflected by increasingly positive net scores. In contrast, both new and consistent binge drinkers failed to demonstrate a shift in behavior on the IGT; they tended to select more cards from the disadvantageous 
Table 2. Demographic characteristics among groups with different drinking trajectories (mean $\pm S D$ )

\begin{tabular}{|c|c|c|c|c|c|c|}
\hline & $\begin{array}{l}\text { Consistent never } \\
\text { drinkers }\end{array}$ & $\begin{array}{l}\text { Occasional } \\
\text { drinkers }\end{array}$ & $\begin{array}{l}\text { New binge } \\
\text { drinkers }\end{array}$ & Quitters & $\begin{array}{c}\text { Consistent binge } \\
\text { drinkers }\end{array}$ & \\
\hline \multicolumn{7}{|l|}{ Time 1 variables } \\
\hline Age & $16.16 \pm 0.56$ & $16.24 \pm 0.54$ & $16.42 \pm 0.75$ & $16.00 \pm 0.50$ & $16.09 \pm 0.70$ & $F_{(4,176)}=1.07 ; p=.37$ \\
\hline \multicolumn{7}{|l|}{ Gender } \\
\hline Female & 33 & 45 & 2 & 7 & 3 & \multirow[t]{2}{*}{$\chi^{2}(4)=11.5 ; p<.05$} \\
\hline Male & 25 & 46 & 10 & 2 & 8 & \\
\hline \multicolumn{7}{|l|}{ School type } \\
\hline Academic & 34 & 53 & 3 & 6 & 3 & \multirow[t]{2}{*}{$\chi^{2}(4)=8.95 ; p=.06$} \\
\hline Vocational & 24 & 38 & 9 & 3 & 8 & \\
\hline Number of drinks & 0 & $1.2 \pm 0.1$ & $1.4 \pm 0.2$ & $3.0 \pm 0.6$ & $3.2 \pm 0.4$ & $F_{(4,176)}=24.7 ; p<.001$ \\
\hline Drinking problems & 0 & $0.4 \pm 0.1$ & $0.8 \pm 0.3$ & $1.0 \pm 0.1$ & $1.4 \pm 0.4$ & $F_{(4,176)}=11.6 ; p<.001$ \\
\hline Working memory & $60.7 \pm 8.1$ & $62.3 \pm 6.3$ & $60.8 \pm 6.9$ & $62.5 \pm 5.2$ & $60.2 \pm 5.4$ & $F_{(4,176)}=0.46 ; p=.68$ \\
\hline Academic performance & $3.5 \pm 1.1$ & $3.4 \pm 1.2$ & $3.6 \pm 1.2$ & $3.5 \pm 1.0$ & $3.5 \pm 1.1$ & $F_{(4,176)}=0.10 ; p=.92$ \\
\hline \multicolumn{7}{|l|}{ Time 2 variables } \\
\hline Number of drinks & 0 & $1.3 \pm 0.1$ & $2.7 \pm 0.4$ & $1.5 \pm 0.5$ & $3.0 \pm 0.4$ & $F_{(4,176)}=21.4 ; p<.001$ \\
\hline Drinking problems & 0 & $0.8 \pm 0.2$ & $2.8 \pm 0.8$ & $0.8 \pm 0.6$ & $4.5 \pm 1.1$ & $F_{(4,176)}=9.6 ; p<.001$ \\
\hline Impulsivity & $100.3 \pm 11.7$ & $102.5 \pm 12.1$ & $108.4 \pm 7.2$ & $106.2 \pm 10.4$ & $110.7 \pm 11.7$ & $F_{(4,176)}=2.45 ; p<.05$ \\
\hline (Lack of) Premeditation & $24.1 \pm 3.8$ & $24.3 \pm 4.5$ & $24.6 \pm 2.7$ & $26.8 \pm 4.5$ & $24.6 \pm 4.2$ & $F_{(4,176)}=1.67 ; p=.16$ \\
\hline Urgency & $22.8 \pm 4.7$ & $23.1 \pm 5.2$ & $27.2 \pm 5.5$ & $23.5 \pm 5.4$ & $27.9 \pm 4.7$ & $F_{(4,176)}=3.89 ; p<.05$ \\
\hline Sensation seeking & $30.1 \pm 6.6$ & $31.7 \pm 6.2$ & $33.3 \pm 3.0$ & $31.2 \pm 5.7$ & $32.2 \pm 8.2$ & $F_{(4,176)}=0.94 ; p=.44$ \\
\hline (Lack of) Perseverance & $23.3 \pm 3.0$ & $23.4 \pm 3.5$ & $23.8 \pm 2.9$ & $24.7 \pm 3.8$ & $23.4 \pm 2.7$ & $F_{(4,176)}=0.86 ; p=.49$ \\
\hline
\end{tabular}

decks. A 4 (group) $\times 5$ (IGT block) ANOVA test revealed a significant block effect after the Greenhouse-Geisser adjustment $\left(F_{(2.8,502.9)}=2.8 ; p<.05\right)$. The interaction effect between group and block was not significant $\left(F_{(211.4,502.9)}=1.3\right.$; $p=.17)$. And there was a significant group effect $\left(F_{(4,176)}=\right.$ 4.42; $p<.001)$. Post hoc Tukey's tests confirmed that both new and consistent binge drinkers performed worse than both never and occasional drinkers $(p<.05)$, and there was no difference in the IGT net scores among consistent never drinkers, occasional drinkers, and quitters.

Figure 2 shows the dot plot of IGT net scores in each group. Previous studies found that $37 \%$ of normal adult con- trols performed disadvantageously on the IGT (i.e., an overall net score of not more than 10), performance within the range of patients with lesions in the ventromedial sector of the prefrontal cortex (VMPC) (Bechara \& Damasio, 2002). Therefore, we used an IGT net score of 10 as a cutoff point. Based on this criterion, $53.4 \%$ of consistent never drinkers and about $67 \%$ of occasional drinkers and $66.7 \%$ of quitters performed less than 10 . In contrast, the vast majority of new $(83.3 \%)$ and consistent $(90.9 \%)$ binge drinkers performed poorly (less than 10) on the IGT. These results demonstrate that the group effect of the IGT performance revealed above is not due to a few extreme cases.

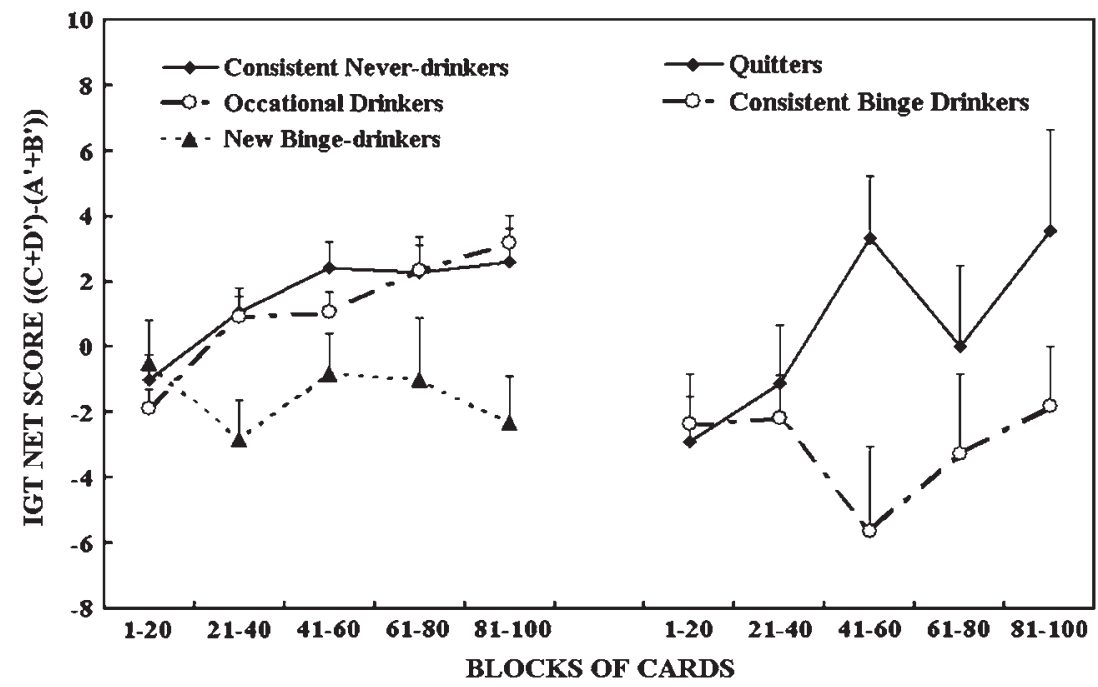

Fig. 1. The IGT net scores $\left[\left(\mathrm{C}^{\prime}+\mathrm{D}^{\prime}\right)-\left(\mathrm{A}^{\prime}+\mathrm{B}^{\prime}\right)\right]$ by group across five blocks of 20 cards expressed as mean $+S E$. Positive net scores reflect advantageous (nonimpaired performance), while negative net scores reflect disadvantageous (impaired) performance. 


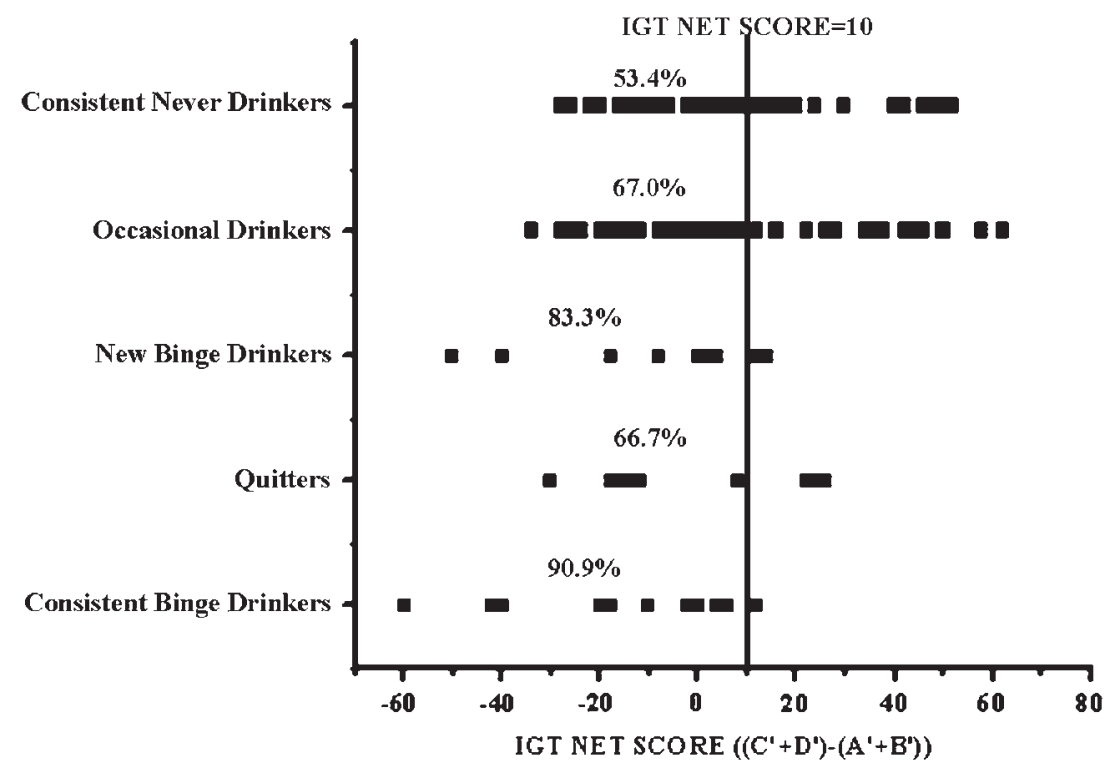

Fig. 2. Dot plot of IGT net scores in each group. Percentage represents the subjects who performed poorly on the IGT (less than the score 10).

\section{Correlations Between Neuropsychological Variables and Impulsivity}

Table 3 reports partial correlations among working memory, IGT net scores, and impulsivity and its four subscales as measured by the UPPS Impulsive Behavior Scale after controlling for gender, age, and school type. The four subscales of impulsivity were significantly correlated with each other (with the exception of nonsignificant correlations between sensation seeking and lack of premeditation and between sensation seeking and lack of perseverance). Working memory significantly correlated with school academic performance $(r=.24 ; p<.001)$. However, working memory did not correlate with any of the subscales of impulsivity. In contrast, IGT net score significantly negatively correlated with impulsivity $(r=-.023 ; p<.001)$. Specifically, the IGT net score significantly negatively correlated with two subscales of impulsivity, urgency and (lack of) perseverance $(r=-.19$, $p<.05 ; r=-.16, p<.05$, respectively). That is, adolescents with lower IGT net score reported higher urgency and (lack of) perseverance 1 year later compared to those with better IGT net scores.

\section{Variables Predicting Drinking Behaviors at Time 2}

We performed hierarchical linear regressions to predict the contribution of IGT at Time 1 to the drinking behaviors at Time 2 one year later. The results of these analyses are presented in Table 4. In order to examine the variance of outcome variables (drinking problems and number of drinks) accounted by the IGT net score, age, gender, school type, baseline value of outcome, working memory capacity, and impulsivity were entered in Step 1. Baseline value of outcome accounted for significant proportion of the variance. In Step 2, adding IGT net score in the model produced a significant increase in the predictive power. IGT net score

Table 3. Partial correlations among neuropsychological functions and impulsivity controlling for age, gender, and school type

\begin{tabular}{lcccccccc}
\hline \hline & 1 & 2 & 3 & 4 & 5 & 6 & 7 & 8 \\
\hline 1. Working memory & - & $.24 * * *$ & .02 & .02 & .03 & -.03 & .11 & .15 \\
2. Academic performance & & - & .06 & -.12 & .05 & -.02 & -.07 & .04 \\
3. IGT net scores & & & - & $-.23 * *$ & -.08 & $-.19 *$ & -.14 & $-.16^{*}$ \\
4. Impulsivity & & & - & $.63 * * *$ & $.71^{* * *}$ & $.66^{* * *}$ & $.61^{* * *}$ \\
5. (Lack of) Premeditation & & & & & - & $.26^{* *}$ & .10 & $.42^{* * *}$ \\
6. Urgency & & & & & - & $.17^{* *}$ & $.20^{* * *}$ \\
7. Sensation seeking & & & & & - & -06 \\
8. (Lack of) Perseverance & & & & & & - & - \\
\hline \hline
\end{tabular}

Note. Results of two-tailed significance tests are denoted by asterisks.

$* p<.05$.

$* * p<.01$.

$* * * p<.001$. 
Table 4. Summary of linear regression analysis for variables predicting drinking problems and number of drinks at Time 2

\begin{tabular}{|c|c|c|c|c|c|c|}
\hline \multirow[b]{2}{*}{ Predictor } & \multicolumn{3}{|c|}{ Drinking problems } & \multicolumn{3}{|c|}{ Number of drinks } \\
\hline & $B$ & $S E B$ & $\beta$ & $B$ & $S E B$ & $\beta$ \\
\hline \multicolumn{7}{|l|}{ Step 1} \\
\hline Age & .09 & .34 & .02 & .02 & .16 & .01 \\
\hline Gender ${ }^{\mathrm{a}}$ & .57 & .4 & .09 & .16 & .19 & .06 \\
\hline School type & .2 & .38 & .02 & .36 & .20 & .13 \\
\hline Baseline value of outcome & .47 & .32 & $.18^{*}$ & .49 & .07 & $.49 * * *$ \\
\hline Impulsivity & .02 & .02 & .08 & .01 & .01 & .10 \\
\hline Working memory & -.01 & .03 & -.02 & .02 & .02 & .08 \\
\hline$R^{2}$ & $.07 *$ & & & $.30 * * *$ & & \\
\hline \multicolumn{7}{|l|}{ Step 2} \\
\hline Age & .08 & .33 & .02 & .07 & .16 & .03 \\
\hline Gender ${ }^{\mathrm{a}}$ & .52 & .38 & .11 & .22 & .18 & .08 \\
\hline School type ${ }^{b}$ & -.01 & .38 & -.01 & .17 & .18 & .07 \\
\hline Baseline value of outcome & .57 & .30 & $.15^{*}$ & .46 & .307 & $.47 * * *$ \\
\hline Impulsivity & .01 & .02 & .03 & .01 & .01 & .05 \\
\hline Working memory & -.00 & .03 & -.02 & .02 & .01 & .10 \\
\hline IGT net score & -.03 & .01 & $-.23 * *$ & -.02 & .01 & $-.15^{*}$ \\
\hline$R^{2}$ & $.11 *$ & & & $.33 * * *$ & & \\
\hline
\end{tabular}

${ }^{a}$ Female as the reference group.

b The vocational school as the reference group.

$* p<.05$.

$* * p<.01$

$* * * p<.001$.

accounted for a significant proportion of the variance. The adolescents with better IGT performance at Time 1 showed fewer drinking problems and consumed fewer drinks per episode at Time 2 compared to those with poor IGT performance $(\beta=-.23, p<.01 ; \beta=-.15, p<.05$, respectively).

\section{DISCUSSION}

Previous longitudinal studies examined the impact of certain neuropsychological functions such as working memory and response inhibitions on initiation of alcohol use behaviors (Aytaclar et al., 1999; Iacono et al., 1999; Nigg et al., 2006; Thush \& Wiers, 2008). Our study extended these studies and, for the first time, investigated one specific neuropsychological function, affective decision making, in the development of alcohol use behaviors. Moreover, most of the previous studies examined brain function in at-risk American adolescents. Our study applied laboratory-based neuropsychological assessments to the normal adolescent population in China, extending the generalizability of the relationship between affective decision making and substance use across cultures.

Previously, we found a synchronous association between IGT performance and IGT (Johnson et al., 2008), suggesting but not establishing a causal relationship between affective decision capacity and binge drinking. The study reported here assessed the prospective nature of this association. As predicted by our hypothesis, those adolescents who progressed to the binge drinking or exhibited consistent binge drinking behaviors performed more poorly on the IGT relative to never and occasional drinkers. Moreover, affective decision making significantly and negatively predicted the number of drinks and drinking problems prospectively independent of previous drinking behaviors, working memory capacity, and impulsivity. The results support our hypothesis that affective decision making can serve as a marker predictive of progression of drinking behaviors among adolescents and indicate that dysfunction in affective decision-making capacity renders youth more vulnerable to compulsive and problem drinking behavior.

A substantial proportion of new (83.3\%) and consistent (90.9\%) binge drinkers performed poorly on the IGT, which demonstrates that the group effect of the poor IGT performance is not due to a few extreme cases. Approximately $53 \%$ of consistent never drinkers and $67 \%$ of occasional drinkers also performed poorly on the IGT. This is consistent with several other reports of poor IGT performance in adolescents (Crone \& van der Molen, 2004; Hooper et al., 2004; Overman et al., 2004). Interestingly, we found that the binge drinkers (new and consistent) even performed significantly worse on the IGT than a subgroup of occasional drinkers who performed poorly (Table A1). These results, together with others from our regression analysis, synergistically demonstrate that although all adolescents might still be undergoing developmental changes in the prefrontal region (i.e., having a "premature" prefrontal cortex) (Giedd, 2004; Gogtay et al., 2004), by and large be mostly susceptible to making suboptimal or "risky" decisions compared to adults, 
there is still individual variability among the adolescents at similar age. And those who are at a higher risk for making bad decisions may potentially and eventually translate into real-life risky behaviors, such as alcoholism and drug dependence.

Two previous cross-sectional studies have reported the relationship between affective decision making and drinking behaviors in adolescents and young adults. Overman et al. (2004) found that polysubstance use was negatively correlated with performance on the IGT analogous task among adolescents. Recently, Goudriaan et al. (2007) examined the relations between decision making measured by the IGT and binge drinking patterns in young adults over a 2-year time period. They found that stable high binge drinkers performed poorly on the IGT compared to the consistent low binge drinkers (Goudriaan et al., 2007). Our results are consistent with these previous studies. Moreover, via the longitudinal study design, our study further supports the notion that a poor capacity for affective decision making is a predisposing factor for alcohol use and abuse.

The prevalence of drinking behaviors in this Chinese sample is similar to that of one large-scale study in China (Xing et al., 2006). The average number of drinking problems reported by the consistent binge drinkers in our study was only 5 (from a list of 23) at Time 2 , suggesting that our study may capture an early stage in progression across abuse trajectories. Interestingly, in our study, the new and consistent binge drinkers both performed poorly on the IGT and scored higher in urgency compared to never and occasional drinkers. Moreover, we found that the IGT net score negatively correlated with urgency. One important difference between urgency and other domains of impulsive behavior is its reliance on emotional factors, and it addresses the role of affect in general and negative affect in particular in the impulsivity behavior (Anestis et al., 2007). Therefore, the results from the neuropsychological task and psychological measures together indicate that adolescents with better decision making may learn to control the drive from their emotions especially the negative emotions better than those with poor decision-making skills. Moreover, those adolescents who are likely to respond imprudently to their negative emotions may increase their propensity to engage in substance use behavior.

Our results suggest that affective decision making and emotion regulation may share the common neural pathways. This notion is supported by previous research suggesting that neural systems subserving affective decision making overlap with those that support emotional intelligence (EI) (Bar-On et al., 2003). In addition, a recent functional magnetic resonance imaging study confirmed that adolescents with high EI responded to emotionally provocative stimuli with less total but more focal brain activation in the brain regions that are critical for affective decision making than did their peers with poorly developed EI (Killgore \& YurgelunTodd, 2007). Taken together, these studies suggest that adolescents with well-developed affective decision making may possess more efficient and effective functioning neural circuitry than do those with poorly developed affective decision making. They might drink recreationally as did the occasional drinkers and quitters in our sample, but their better affective decision-making capacity protects them from abusive drinking. However, since behavioral tests are not sufficient to identify the brain's involvement with specificity, further research using imaging methods should address differences between brain circuitry underlying affective decision making in those adolescents who progress toward greater substance abuse compared to those who do not.

Since prior studies have shown that working memory is highly related to general cognitive functions such as reading, mathematics, and reasoning (Colom et al., 2004; Jarrold \& Towse, 2006), it is not surprising to see that working memory was highly associated with school academic performance in this study. However, neither working memory nor academic performance correlated to any subscales of impulsivity. These results are consistent with prior observations that working memory tasks activate the DLPC-related neural circuitry associated with "cold" cognitive systems (Funahashi, 2006). Moreover, we found that neither working memory nor academic performance differed in adolescents with various drinking trajectories. Our results suggest that affective decision-making impairment in binge drinkers may be independent of the type of general cognitive intelligence impairment, most likely revealed by tests of working memory capacity (i.e., fluid intelligence).

A limitation of the current study is reliance on self-reports of binge drinking, raising the question of whether a culturally driven self-presentation bias may have influenced the results. A recent study found a binge drinking prevalence rate of $13.2 \%$ in the 32,425 senior high school students across 18 provinces of China (Xing et al., 2006) comparable to those found in our study, $11 \%$ at 10th grade and $12.7 \%$ at 11th grade. Alcohol use in Western students differs significantly across racial/ethnic groups (Wallace et al., 2002; Wonga et al., 2004). Although the rate of alcohol use in our study is lower than the overall rate of U.S. sample, it is comparable to that of Asian students reported in the United States both national and regional studies (Wallace et al., 2002; Wonga et al., 2004). The rate of alcohol use is also similar to that of high school students in Canada (Feldman et al., 1999). Moreover, empirical studies have also shown that the selfreported data are, by and large, valid across racial, ethnic, and cultural groups (Johnston et al., 1994; Wallace \& Bachman, 1993). Therefore, it is unlikely that there is cultural bias to underreport the socially undesirable behavior. Furthermore, students with low IGT scores may have been more prone to report binge drinking compared with those with high IGT scores, regardless of actual drinking behavior. However, if there is a social desirability self-presentation bias in favoring those with high IGT scores, this bias should be reflected in other socially undesirable behavior as well, such as their poor school academic performance. However, students with low IGT scores did not self-report poorer academic performance than those with high IGT scores (Tables 1 and 3). Taken together, it is unlikely that our findings were greatly 
affected by culturally or neurocognitively driven selfpresentation bias.

There are other limitations in this study. First, the sample size of adolescents with substance use is relatively small, thus limiting the power to detect gender effects in the current study. And the prevalence of drinkers did not differ significantly from Time 1 to Time 2 (Table A2). However, the prevalence of substance use in our sample was very similar to that of other large-scale population studies of students in China (Xing et al., 2006). Second, our study included students from both major types of Chinese high schools, which suggests that the findings are widely generalizable to Chinese youth. Future studies are needed to establish reliability to other cultural/environmental settings. Third, the longitudinal design of the present research covered only 1 year, so we do not know if the binge drinkers in our study would eventually progress to even more problematic drinking behavior. However, previous research showed that substance use behaviors among adolescents significantly predict their subsequent substance use in adulthood (Johnson et al., 2000). Nevertheless, to our knowledge, this study is among the first to identify neuropsychological risk factors for substance use behaviors among a general adolescent population. Moreover, the longitudinal design provides evidence directly supporting the notion that the specific neuropsychological function, affective decision making, could serve as a unique marker to predict escalated alcohol use among adolescents.

\section{ACKNOWLEDGMENTS}

This research was supported by the University of Southern California Pacific Rim Transdisciplinary Tobacco and Alcohol Use Research Center, funded by the National Cancer Institute (grant \#1 P50 CA84735-02 to C.A.J.) and the National Institute on Drug Abuse (grant \#DA16708 to A.B. and DA 16094 to A.W.S.). The authors thank Peggy Gallaher, Steven Cen, Joel Milam, Qian Guo, Kari-Lyn Kobayakawa Sakuma, and Janet Okamoto for their contribution to this project. We also thank Qiong Wang, Rong Lu, Jiang Miao, Yi Yuan, and Jingfu Shi for their assistance with project coordination and data collection. Finally, we express our gratitude to the Municipal Government, Health Bureau, and Education Committee in Chengdu, China, for their support. The authors declare no competing financial interests.

\section{REFERENCES}

Anestis, M.D., Selby, E.A., \& Joiner, T.E. (2007). The role of urgency in maladaptive behaviors. Behaviour Research and Therapy, 45(12), 3018-3029.

Aytaclar, S., Tarter, R.E., Kirisci, L., \& Lu, S. (1999). Association between hyperactivity and executive cognitive functioning in childhood and substance use in early adolescence. Journal of the American Academy of Child and Adolescent Psychiatry, 38(2), 172-178.

Bar-On, R., Tranel, D., Denburg, N.L., \& Bechara, A. (2003). Exploring the neurological substrate of emotional and social intelligence. Brain, 126(Pt 8), 1790-1800.
Bechara, A. (2004). The role of emotion in decision-making: Evidence from neurological patients with orbitofrontal damage. Brain Cognition, 55(1), 30-40.

Bechara, A., Damasio, A.R., Damasio, H., \& Anderson, S.W. (1994). Insensitivity to future consequences following damage to human prefrontal cortex. Cognition, 50(1-3), 7-15.

Bechara, A. \& Damasio, H. (2002). Decision-making and addiction (part I): Impaired activation of somatic states in substance dependent individuals when pondering decisions with negative future consequences. Neuropsychologia, 40(10), 1675-1689.

Bechara, A., Tranel, D., Damasio, H., \& Damasio, A.R. (1996). Failure to respond autonomically to anticipated future outcomes following damage to prefrontal cortex. Cerebral Cortex, 6(2), 215-225.

Brown, S.A., McGue, M., Maggs, J., Schulenberg, J., Hingson, R., Swartzwelder, S., Martin, C., Chung, T., Tapert, S.F., Sher, K., Winters, K.C., Lowman, C., \& Murphy, S. (2008). A developmental perspective on alcohol and youths 16 to 20 years of age. Pediatrics, 121, S290-S310.

Centers for Disease Control and Prevention. (1999). Behavioral risk factor surveillance system survey questionnaire. Atlanta, GA: US Department of Health and Human Services, Centers for Disease Control and Prevention.

Chaytor, N. \& Schmitter-Edgecombe, M. (2004). Working memory and aging: A cross-sectional and longitudinal analysis using a self-ordered pointing task. Journal of the International Neuropsychological Society, 10(4), 489-503.

Colom, R., Rebello, I., Palacios, A., Juan-Espinosa, M., \& Kyllonen, P.C. (2004). Working memory is (almost) perfectly predicted by g. Intelligence, 32, 277-296.

Crone, E.A. \& van der Molen, M.W. (2004). Developmental changes in real life decision making: Performance on a gambling task previously shown to depend on the ventromedial prefrontal cortex. Developmental Neuropsychology, 25(3), 251-279.

Feldman, L., Harvey, B., Holowaty, P., \& Shortt, L. (1999). Alcohol use beliefs and behaviors among high school students. The Journal of Adolescent Health, 24(1), 48-58.

Funahashi, S. (2006). Prefrontal cortex and working memory processes. Neuroscience, 139(1), 251-261.

Giedd, J.N. (2004). Structural magnetic resonance imaging of the adolescent brain. Annals of the New York Academy of Sciences, 1021, 77-85.

Gogtay, N., Giedd, J.N., Lusk, L., Hayashi, K.M., Greenstein, D., Vaituzis, A.C., Nugent, T.F.III, Herman, D.H., Clasen, L.S., Toga, A.W., Rapoport, J.L., \& Thompson, P.M. (2004). Dynamic mapping of human cortical development during childhood through early adulthood. Proceedings of the National Academy of Sciences of the United States of America, 101(21), 8174-8179.

Goudriaan, A.E., Grekin, E.R., \& Sher, K.J. (2007). Decision-making and binge-drinking: A longitudinal study. Alcoholism, Clinical and Experimental Research, 31(6), 928-938.

Grenard, J.L., Ames, S.L., Wiers, R.W., Thush, C., Sussman, S., \& Stacy, A.W. (2008). Working memory capacity moderates the predictive effects of drug-related associations on substance use. Psychology of Addictive Behaviors, 22(3), 426-432.

Hooper, C.J., Luciana, M., Conklin, H.M., \& Yarger, R.S. (2004). Adolescents' performance on the Iowa Gambling Task: Implications for the development of decision making and ventromedial prefrontal cortex. Developmental Psychology, 40(6), 1148-1158. 
Iacono, W.G., Carlson, S.R., Taylor, J., Elkins, I.J., \& McGue, M. (1999). Behavioral disinhibition and the development of substance-use disorders: Findings from the Minnesota Twin Family Study. Development and Psychopathology, 11(4), 869-900.

Jarrold, C. \& Towse, J.N. (2006). Individual differences in working memory. Neuroscience, 139(1), 39-50.

Johnson, A.C., Palmer, P.H., Chou, C.P., Pang, Z., Zhou, D., Dong, L., Xiang, H., Yang, P., Xu, H., Wang, J., Fu, X., Guo, Q., Sun, P., Ma, H., Gallaher, P.E., Xie, B., Lee, L., Fang, T., \& Unger, J.B. (2006). Tobacco use among youth and adults in Mainland China: The China Seven Cities Study. Public Health, 120(12), $1156-1169$.

Johnson, C.A., Xiao, L., Palmer, P., Sun, P., Wang, Q., Wei, Y., Jia, Y., Grenard, J.L., Stacy, A.W., \& Bechara, A. (2008). Affective decision-making deficits, linked to a dysfunctional ventromedial prefrontal cortex, revealed in 10th grade Chinese adolescent binge drinkers. Neuropsychologia, 46(2), 714-726.

Johnson, P., Boles, S., \& Kleber, H. (2000). The relationship between adolescent smoking and drinking and likelihood estimates of illicit drug use. Journal of Addictive Diseases, 19(2), 75-81.

Johnston, L.D., Driessen, F.M.H.M., \& Kokkevi, A. (1994). Surveying student drug misuse: A six-country pilot study. Strasbourg, France: Council of Europe.

Kerr, A. \& Zelazo, P.D. (2004). Development of "hot" executive function: The children's gambling task. Brain Cognition, 55(1), $148-157$.

Killgore, W.D. \& Yurgelun-Todd, D.A. (2007). Neural correlates of emotional intelligence in adolescent children. Cognitive, Affective \& Behavioral Neuroscience, 7(2), 140-151.

Kolbe, L.J., Kann, L., \& Collins, J.L. (1993). Overview of the Youth Risk Behavior Surveillance System. Public Health Reports, 108(Suppl 1), 2-10.

Nigg, J.T., Wong, M.M., Martel, M.M., Jester, J.M., Puttler, L.I., Glass, J.M., Adams, K.M., Fitzgerald, H.E., \& Zucker, R.A. (2006). Poor response inhibition as a predictor of problem drinking and illicit drug use in adolescents at risk for alcoholism and other substance use disorders. Journal of the American Academy of Child and Adolescent Psychiatry, 45(4), 468-475.

O’Doherty, J., Kringelbach, M.L., Rolls, E.T., Hornak, J., \& Andrews, C. (2001). Abstract reward and punishment representations in the human orbitofrontal cortex. Nature Neuroscience, 4(1), 95-102.

Overman, W.H., Frassrand, K., Ansel, S., Trawalter, S., Bies, B., \& Redmond, A. (2004). Performance on the IOWA card task by adolescents and adults. Neuropsychologia, 42(13), 1838-1851.

Oya, H., Adolphs, R., Kawasaki, H., Bechara, A., Damasio, A., \& Howard, M.A.III. (2005). Electrophysiological correlates of reward prediction error recorded in the human prefrontal cortex. Proceedings of the National Academy of Sciences of the United States of America, 102(23), 8351-8356.
Perry, W., Heaton, R.K., Potterat, E., Roebuck, T., Minassian, A., \& Braff, D.L. (2001). Working memory in schizophrenia: Transient "online" storage versus executive functioning. Schizophrenia Bulletin, 27(1), 157-176.

Peterson, J.B., Pihl, R.O., Higgins, D., \& Lee, A. (2002). Neurocognitive battery (version 2.0). Deerfield Beach, FL: Examcorp.

Petrides, M. (1995). Impairments on nonspatial self-ordered and externally ordered working memory tasks after lesions of the mid-dorsal part of the lateral frontal cortex in the monkey. The Journal of Neuroscience, 15(1 Pt 1), 359-375.

Petrides, M., Alivisatos, B., Meyer, E., \& Evans, A.C. (1993). Functional activation of the human frontal cortex during the performance of verbal working memory tasks. Proceedings of the National Academy of Sciences of the United States of America, 90(3), 878-882.

Petrides, M. \& Milner, B. (1982). Deficits on subject-ordered tasks after frontal- and temporal-lobe lesions in man. Neuropsychologia, 20(3), 249-262.

Stocco, A. \& Fum, D. (2008). Implicit emotional biases in decision making: The case of the Iowa Gambling Task. Brain and Cognition, 66(3), 253-259.

Thush, C., Wiers, R.W., Ames, S.L., Grenard, J.L., Sussman, S., \& Stacy, A.W. (2008). The interactions between implicit and explicit cognition and working memory capacity in the prediction of alcohol use in at-risk adolescents. Drug and Alcohol Dependence, 94(1-3), 116-124.

Wagar, B.M. \& Dixon, M. (2006). Affective guidance in the Iowa gambling task. Cognitive, Affective \& Behavioral Neuroscience, 6(4), 277-290.

Wallace, J.M. Jr. \& Bachman, J.G., (1993). Validity of self-reports in student-based studies on minority populations: Issues and concerns. NIDA Research Monograph, 130, 167-200.

Wallace, J.M. Jr., Bachman, J.G., O'Malley, P.M., Johnston, L.D., Schulenberg, J.E., \& Cooper, S.M. (2002). Tobacco, alcohol, and illicit drug use: Racial and ethnic differences among U.S. high school seniors, 1976-2000. Public Health Reports, 117(Suppl 1), S67-S75.

White, H.R. \& Labouvie, E.W. (1989). Towards the assessment of adolescent problem drinking. Journal of Studies on Alcohol, 50(1), 30-37.

Whiteside, S.P. \& Lynam, D.R. (2001). The five factor model and impulsivity: Using a structural model of personality to understand impulsivity. Personality and Individual Differences, 30, 669-689.

Wonga, M.M., Klingleb, R.S., \& Price, R.K. (2004). Alcohol, tobacco, and other drug use among Asian American and Pacific Islander adolescents in California and Hawaii. Addictive Behavior, 29, 127-141.

Xing, Y., Ji, C., \& Zhang, L. (2006). Relationship of binge drinking and other health-compromising behaviors among urban adolescents in China. Journal of Adolescent Health, 39(4), 495-500. 


\section{APPENDIX}

Table A1. Measures between occasional drinkers who performed poorly (less than the score 10) on the IGT and binge drinkers (new and consistent) (mean $\pm S D$ )

\begin{tabular}{|c|c|c|c|}
\hline & Occasional drinkers & $\begin{array}{c}\text { Binge drinkers } \\
\text { (new and consistent) }\end{array}$ & \\
\hline$N$ & 61 & 23 & \\
\hline \multicolumn{4}{|l|}{ Time 1 variables } \\
\hline Age & $16.18 \pm 0.53$ & $16.20 \pm 0.75$ & $t_{(82)}=0.46 ; p=.64$ \\
\hline \multicolumn{4}{|l|}{ Gender } \\
\hline Female & 30 & 5 & \multirow[t]{2}{*}{$\chi_{(1)}^{2}=5.17 ; p<.05$} \\
\hline Male & 31 & 18 & \\
\hline \multicolumn{4}{|l|}{ School type } \\
\hline Academic & 33 & 6 & \multirow[t]{2}{*}{$\chi_{(1)}^{2}=5.26 ; p<.05$} \\
\hline Vocational & 28 & 17 & \\
\hline Number of drinks & $1.38 \pm 1.0$ & $2.53 \pm 1.47$ & $t_{(82)}=4.43 ; p<.001$ \\
\hline Drinking problems & $0.45 \pm 0.70$ & $1.26 \pm 1.19$ & $t_{(82)}=4.99 ; p<.001$ \\
\hline Working memory & $62.7 \pm 6.8$ & $60.3 \pm 7.5$ & $t_{(82)}=1.47 ; p=.15$ \\
\hline Academic performance & $3.56 \pm 1.1$ & $3.57 \pm 1.1$ & $t_{(82)}=0.04 ; p=.82$ \\
\hline IGT net score & $-2.0 \pm 10.1$ & $-11.3 \pm 20.8$ & $t_{(82)}=2.88 ; p<.05$ \\
\hline \multicolumn{4}{|l|}{ Time 2 variables } \\
\hline Number of drinks & $1.41 \pm 1.2$ & $2.8 \pm 1.3$ & $t_{(82)}=5.17 ; p<.001$ \\
\hline Drinking problems & $0.97 \pm 2.5$ & $3.58 \pm 4.8$ & $t_{(82)}=3.06 ; p<.05$ \\
\hline Impulsivity & $103.7 \pm 11.7$ & $109.1 \pm 9.3$ & $t_{(82)}=1.81 ; p=.07$ \\
\hline (Lack of) Premeditation & $24.2 \pm 4.5$ & $24.6 \pm 3.0$ & $t_{(82)}=0.11 ; p=.91$ \\
\hline Urgency & $23.7 \pm 5.3$ & $27.5 \pm 4.3$ & $t_{(82)}=3.35 ; p<.05$ \\
\hline Sensation seeking & $31.9 \pm 5.8$ & $32.7 \pm 6.5$ & $t_{(82)}=0.87 ; p=.36$ \\
\hline (Lack of) Perseverance & $23.4 \pm 3.5$ & $23.6 \pm 2.7$ & $t_{(82)}=0.19 ; p=.82$ \\
\hline
\end{tabular}

Note. The table shows the comparison between occasional drinkers who performed poorly (less than the score 10) on the IGT, and the binge drinkers (new and consistent). Chi-square tests show that the percentage of males and vocational students was significantly higher among binge drinkers compared to that of occasional drinkers $\left(\chi_{(1)}^{2}=5.17 ; p<.05 ; \chi_{(1)}^{2}=5.26 ; p<.05\right.$, respectively). Independent $t$ tests revealed that binge drinkers had significantly more drinking behaviors (number of drinks and drinking problems) than this subgroup of occasional drinkers at both years. Moreover, they performed significantly worse on the IGT and scored higher on urgency than those occasional drinkers $\left(t_{(82)}=2.88 ; p<.05 ; t_{(82)}=3.35 ; p<.05\right.$, respectively). Such significant results still stood after controlling for gender and school type. However, the two groups did not differ in other measures in terms of working memory, school academic performance, lack of premeditation, sensation seeking, and lack of perseverance. Results of two-tailed significance tests are denoted in boldface.

Table A2. Drinkers and drinking behaviors in 2 years $(N=181)$

\begin{tabular}{lccr}
\hline \hline & Baseline & Year 1 & \\
\hline Never drinkers & $39.8(72)$ & $32.0(58)$ & $\chi^{2}(2)=2.36 ; p=.32$ \\
Occasional drinkers (ever/past 30 days) & $49.2(89)$ & $55.2(100)$ & \\
Binge drinkers & $11.0(20)$ & $12.7(23)$ & $t_{(180)}=0.06 ; p=.96$ \\
Number of drinks & 1.16 & 1.19 & $t_{(180)}=3.15 ; p<.05$ \\
Drinking problems & 0.3 & 0.87 & \\
\hline \hline
\end{tabular}

